\title{
ON THE POWERS OF A REAL NUMBER REDUCED MODULO ONE
}

\author{
BY \\ FRED SUPNICK, H. J. COHEN AND J. F. KESTON
}

1. Introduction. Let us consider the sequence

$$
\alpha-[\alpha], \quad \alpha^{2}-\left[\alpha^{2}\right], \quad \alpha^{3}-\left[\alpha^{3}\right], \cdots,
$$

where $\alpha$ is a real number greater than one ( $[x]$ denotes the greatest integer $\left.{ }^{1}\right)$ less than or equal to $x$ ). It has been shown by Koksma (cf. [1]) that the terms of $(1.1)$ distribute uniformly on the interval $(0,1)$ for almost all $\alpha>1$. We note, however, that the elements of (1.1) need not be distinct (e.g. $\alpha$ integral, or $\alpha=2^{1 / 2}$ ).

Consider all the values $v_{1}, v_{2}, v_{3}, \cdots\left(v_{i} \neq v_{j}\right.$ for $\left.i \neq j\right)$ assumed at least once by the terms of (1.1). Let us denote the set of all positive integers $i$ such that $\alpha^{i}-\left[\alpha^{i}\right]=v_{1}$ by $C_{1}$, the set of all positive integers $i$ such that $\alpha^{i}-\left[\alpha^{i}\right]=v_{2}$ by $C_{2}$, etc. That is, the set $g:\{1,2, \cdots, n, \cdots\}$ partitions into sets $C_{1}, C_{2}, \cdots$ :

$$
\mathrm{g}=C_{1}+C_{2}+C_{3}+\cdots
$$

with the property that $j, k \in C_{\nu}$ if and only if

$$
\alpha^{j}-\left[\alpha^{j}\right]=\alpha^{k}-\left[\alpha^{k}\right]
$$

i.e. if and only if

$$
\alpha^{k}-\alpha^{3}=r,
$$

$r$ integral. The set $\left\{C_{1}, C_{2}, C_{3}, \cdots\right\}$ will be denoted by $g / \alpha$, and will be called the decomposition of $g$ induced by $\alpha$.

In this paper we study the decomposition $g / \alpha$ for $\alpha>1$.

The elements $C_{\nu}$ of $g / \alpha$ will be called exponent classes. If an exponent class contains only one element of $g$, it will be called unitary; if each $C_{\boldsymbol{v}}$ is unitary, then $g / \alpha$ will be referred to as a unitary decomposition.

$g / \alpha$ is unitary if and only if the equation (1.3) has no solutions in positive integers $j, k, r$. Thus if $\alpha$ is not an algebraic integer, the decomposition is unitary (cf. [2]). Therefore we consider only integral algebraic $\alpha$.

If $\alpha$ is a rational integer, the problem is trivial. Therefore we consider only irrational integral algebraic $\alpha$.

Let the minimal polynomial of $\alpha$ be

Presented to the Society, October 26, 1957; received by the editors February 25, 1959.

(1) The term "integer" not preceded by "algebraic" will mean "rational integer." 


$$
M_{\alpha}(x)=x^{n}+a_{1} x^{n-1}+a_{2} x^{n-2}+\cdots+a_{n-1} x+a_{n},
$$

where the $a_{i}$ are integers. If $a_{n}$ is positive, $M_{\alpha}(x)$ has at least one positive zero other than $\alpha$ and hence $M_{\alpha}(x)$ cannot divide any polynomial of the form $x^{k}-x^{j}-r$, where $j, k, r$ are positive integers with $j$ less than $k$ (since any polynomial of this form has only one positive zero). Thus, if $a_{n}$ is positive, $\alpha$ cannot satisfy any relation of the form (1.3) and so the decomposition is unitary. Therefore we consider only irrational integral algebraic $\alpha$ whose minimal polynomial has a negative constant term.

We let $L(\alpha)$ denote the number of nonzero terms in $M_{\alpha}(x)$.

If an exponent class contains exactly two elements, it will be called binary.

Summary of main results. For $L(\alpha)=2,3$ the complete decompositions $g / \alpha$ are obtained (cf. Theorems 1 and $7, \S 2$ ). For $L(\alpha) \geqq 3$, we prove (i) that each $C_{\nu}$ is either unitary or binary, and (ii) that at most a finite number of the $C_{\nu}$ are binary (cf. Theorems 2 and $3, \S 2$ ). Sufficient conditions for unitary decomposition are obtained in Corollary 5.1 and Theorems 4, 5 and 6 (cf. \$2).

2. Statement of results. In the following theorems $\alpha$ is understood to be a real irrational algebraic integer greater than unity whose minimal polynomial has a negative constant term.

TheOREM 1. Suppose $L(\alpha)=2$; that is, $M_{\alpha}(x)=x^{n}-K$, where $K>0$. Then the set $\{n, 2 n, 3 n, \cdots\}$ comprises a single exponent class $C_{\nu_{0}}$ of $\mathfrak{g} / \alpha$, while each positive integer not belonging to $C_{\nu_{0}}$ forms a unitary exponent class (cf. §3).

Theorem 2. If $L(\alpha) \geqq 3$, then no $C_{\nu}$ can contain more than two elements $(c f . \S 4)$.

Lemma $5.1\left(^{2}\right)$. Let $L(\alpha) \geqq 3$, and suppose that $(j, k)$ is a binary exponent class of $g / \alpha$, where $j<k$. Then,

$$
0<\frac{\alpha^{n}-\left|a_{n}\right|}{\alpha^{n}} \leqq \frac{1}{\alpha^{k-j}},
$$

where $a_{n}$ denotes the (negative) constant term of $M_{\alpha}(x)$ (cf. §5).

Corollary 5.1. Let $L(\alpha) \geqq 3$, and suppose that $a_{i} \geqq 0$ in $M_{\alpha}(x)$, for $1 \leqq i$ $\leqq n-1$. Then $\mathrm{g} / \alpha$ is unitary.

Corollary 5.2. Let $L(\alpha) \geqq 3$, and suppose that $g / \alpha$ is nonunitary. Then,

$$
\alpha^{n-1}(\alpha-1) \leqq\left|a_{n}\right|<\alpha^{n} .
$$

COROLlaRy 5.3. Let $L(\alpha) \geqq 3$, and suppose that $g / \alpha$ is nonunitary. Let the roots of $M_{\alpha}(x)$ be denoted by $z_{1}, z_{2}, \cdots, z_{n}$. Then,

(2) Integral parts of lemma-numbers indicate sections containing proofs; corollary-numbers have same integral parts as the theorem or lemma to which they are attached. 


$$
\alpha-1 \leqq\left|z_{i}\right| \leqq \alpha \quad(i=1,2, \cdots, n) .
$$

TheOREM 3. For any $g / \alpha$, at most a finite number of the $C_{\nu}$ are binary (cf. $\S 6)$.

We note that Theorems 1,2 and 3 jointly imply the following statement: Let $\alpha$ be a real number greater than one. Then, the equation $\alpha^{x}-\alpha^{y}=z$ has at most a finite number of solutions in positive integers $x, y, z$, except in the case when $\alpha=K^{1 / n}$, where $n, K$ are positive integers $\left({ }^{3}\right)$.

Theorems 4, 5 and 6 state sufficient conditions for unitary decomposition.

Theorem 4. Suppose

$$
M_{\alpha}(x)=x^{n}-b_{1} x^{n-1}-b_{2} x^{n-2}-\cdots-b_{n-1} x-b_{n},
$$

where each $b_{i} \geqq 0$, and $\sum_{i=1}^{n-1} b_{i}>1$. Then $g / \alpha$ is unitary (cf. $\S 7$ ).

CoROllary 4.1. Let $M_{\alpha}(x)$ be of form (2.4) where each $b_{i} \geqq 0$. Then, if $L(\alpha) \geqq 4, g / \alpha$ is unitary.

Theorem 5. Suppose $M_{\alpha}(x)$ is of the form

$$
M_{\alpha}(x)=x^{n}+a_{1} x^{n-1}+a_{2} x^{n-2}+\cdots+a_{n-1} x-1,
$$

where $\sum_{i=1}^{n-1} a_{i} \neq-1$. Then $g / \alpha$ is unitary (cf. §8).

THEOREM 6. If $M_{\alpha}(x)$ has two real roots of the same sign, then $g / \alpha$ is unitary (cf. $\S 9)$.

Theorem 7 states the complete decomposition for the case $L(\alpha)=3$.

TheOREM 7. Let $M_{\alpha}(x)=x^{n}+a x^{n-r}-K,(a \neq 0, K>0,0<r<n)$.

(a) If $a \neq-1$, then $g / \alpha$ is unitary.

(b) If $a=-1$, then the integers $(n-r, n)$ form a binary class, by definition. There will be no other binary classes, unless $M_{\alpha}(x)$ is of the special form:

$$
M_{\alpha}(x)=x^{3 t}-x^{t}-1 .
$$

In this exceptional case, each of the pairs $(t, 3 t)$ and $(4 t, 5 t)$ forms a binary class, and there are no others ( $c f . \$ 10)$.

3. Proof of Theorem 1. We assume $n>1$. The integers $(n, 2 n, 3 n, \cdots)$ clearly belong to the same exponent class $C_{v_{0}}$. If $m=a n+b$, where $a \geqq 0$ and $0<b<n$, then $m$ cannot belong to $C_{v_{0}}$. Otherwise, we would have

$$
\alpha^{a n+b}-\alpha^{n}=t
$$

where $t$ is integral; but this becomes

(3) A theorem of A. Gelfond (cf. [3]) contains, as a special case, an analogous result for the closely related equation $\alpha^{x}-\alpha^{y}=\delta^{z}$, where $\alpha, \delta$ are given real, algebraic numbers. 


$$
K^{a} \alpha^{b}-K=t,
$$

which is of lower degree in $\alpha$ than the degree $n$ of $M_{\alpha}(x)$. Thus the integers $(n, 2 n, 3 n, \cdots)$ comprise the complete class $C_{v_{0}}$.

Suppose

$$
r=a_{1} n+b_{1}, \quad s=a_{2} n+b_{2},
$$

where $a_{i} \geqq 0$ and $0<b_{i}<n,(i=1,2)$, and assume that $r$ and $s$ were in the same class. We would then have

$$
\alpha^{a_{1} n+b_{1}}-\alpha^{a_{2} n+b_{2}}=t
$$

or,

$$
K^{a_{1}} \alpha^{b_{1}}-K^{a_{2}} \alpha^{b_{2}}-t=0,
$$

which, as before, is impossible unless $r$ equals $s$.

4. Proof of Theorem 2. We first prove

LEMMA 4.1. If $M_{\alpha}(x)$ has a root $\beta$ such that $|\beta|<1$, then no exponent class in $\mathrm{g} / \alpha$ can contain more than two elements.

Proof. Suppose the integers $j, k$ belong to the same exponent class, where $j<k$. That is,

$$
\alpha^{k}-\alpha^{j}=r,
$$

where $r$ is a positive integer. Then, since $M_{\alpha}(x)$ must divide $x^{k}-x^{j}-r$,

$$
\beta^{k}-\beta^{j}=r \text {. }
$$

Therefore

$$
r \leqq|\beta|^{k}+|\beta|^{j}<2
$$

Hence $r=1$. Thus, if some exponent class contained three elements $u<v<w$, we would then have

$$
\alpha^{w}-\alpha^{u}=1,
$$

and

$$
\alpha^{w 0}-\alpha^{v}=1 .
$$

But these relations imply $\alpha^{u}=\alpha^{v}$, which is impossible.

Proof of Theorem 2. We now assume, in view of Lemma 4.1, that no root of $M_{\alpha}(x)$ has absolute value less than unity.

If the theorem were false, there would exist three positive integers $j<k<m$, such that

$$
\alpha^{k}-\alpha^{j}=r
$$

and

$$
\alpha^{m}-\alpha^{j}=s,
$$


where $r, s$ are positive integers, $r<s$. Let us write the $n$ roots $z_{1}, z_{2}, \cdots, z_{n}$ of $M_{\alpha}(x)$ in the form

$$
z_{\nu}=\rho_{\nu} e^{i \theta_{\nu}}, \quad(\nu=1,2, \cdots, n),
$$

where each $\rho_{\nu} \geqq 1$.

Now from (4.1),

$$
x^{k}-x^{3}-r=M_{\alpha}(x) \cdot P(x),
$$

where $P(x)$ is a polynomial with integral coefficients. We note that if $|x|>\alpha$, then

$$
\left|x^{k}-x^{i}\right| \geqq|x|^{k}-|x|^{i}>\alpha^{k}-\alpha^{j}=r ;
$$

thus no zero of $x^{k}-x^{i}-r$ has absolute value greater than $\alpha$. Since the left member of (4.4) has each $z$, among its roots, then

$$
1 \leqq \rho_{\nu} \leqq \alpha, \quad(\nu=1,2, \cdots, n) .
$$

We will now prove that each $\rho_{\nu}=\alpha,(\nu=1,2, \cdots, n)$. Substituting (4.3) into (4.4), we have

$$
\begin{aligned}
& r=\rho_{\nu}^{k} \cos k \theta_{\nu}-\rho_{\nu}^{j} \cos j \theta_{\nu}, \\
& 0={ }_{\rho_{\nu}}^{k} \sin k \theta_{\nu}-\rho_{\nu}^{j} \sin j \theta_{\nu} .
\end{aligned}
$$

Transposing the second term of each right member to the left, squaring each equation, and adding, we obtain

$$
r^{2}+2 r \rho_{\nu}^{j} \cos j \theta_{\nu}+\rho_{\nu}^{2 j}=\stackrel{2 k}{\rho_{\nu}} .
$$

Similarly, from (4.2),

$$
s^{2}+2 s \rho_{\nu}^{j} \cos j \theta_{\nu}+\rho_{\nu}^{2 j}=\rho_{\nu}^{2 m} .
$$

Eliminating $\theta_{\nu}$ between (4.7) and (4.8),

$$
r^{2} s-s^{2} r+s \rho_{\nu}^{2 j}-r \rho_{v}^{2 j}=s \rho_{\nu}^{2 k}-r \rho_{\nu}^{2 m} .
$$

Thus, defining the polynomial

$$
G(x)=r x^{2 m}-s x^{2 k}+(s-r) x^{2 j}-r s(s-r),
$$

we have

$$
G\left(\rho_{v}\right)=0, \quad(\nu=1,2, \cdots, n) .
$$

From (4.9), using (4.1) and (4.2), we obtain

$$
G(0)=G(1)=G\left(\alpha^{1 / 2}\right)=-r s(s-r)<0 .
$$


Therefore, there exist $x_{0}, x_{1}, 0<x_{0}<1,1<x_{1}<\alpha^{1 / 2}$, such that

$$
G^{\prime}\left(x_{0}\right)=G^{\prime}\left(x_{1}\right)=0 \text {. }
$$

But, since $G^{\prime}(x)$ is a trinomial, it has at most two positive roots, which must then be $x_{0}, x_{1}$. Moreover, since the leading coefficient of $G^{\prime}(x)$ is positive, we have

$$
G^{\prime}(x)>0 \quad \text { for } x>x_{1} .
$$

That is, $G(x)$ is strictly increasing for $x>x_{1}$. But $G(\alpha)=0$, and $x_{1}<\alpha$. Therefore, $G(x)<0$ for $x_{1} \leqq x<\alpha$. We now show that $G(x)<0$ for $1<x<x_{1}$. Assume that $G\left(x_{2}\right) \geqq 0$, where $1<x_{2}<x_{1}$. Since $G(1)$ and $G\left(x_{1}\right)$ are each negative, this would imply that $G^{\prime}(x)$ has a root between 1 and $x_{1}$, which is impossible. We thus have

$$
G(x)<0 \quad \text { for } 1 \leqq x<\alpha .
$$

Therefore, from (4.11), (4.10), and (4.6), we conclude

$$
\rho_{\nu}=\alpha, \quad(\nu=1,2, \cdots, n) .
$$

Now, taking the product of the absolute values of the roots (4.3) of $M_{\alpha}(x)$, we obtain

$$
\alpha^{n}=\left|a_{n}\right|,
$$

which contradicts the assumption that $L(\alpha) \geqq 3$. This completes the proof of Theorem 2.

5. Proof of Lemma 5.1. Let $\alpha^{k}-\alpha^{i}=r$. Then

$$
x^{k}-x^{j}-r=M_{\alpha}(x) \cdot P(x),
$$

where $P(x)$ is a polynomial of degree $(k-n)$, having integral coefficients. Denote the roots of $M_{\alpha}(x)$ by $z_{1}, z_{2}, \cdots, z_{n}$, and those of $P(x)$ by $\omega_{1}, \omega_{2}, \cdots$, $\omega_{k-n}$. Since the left member of (5.1) has each $z_{i}$ among its roots and $\alpha$ as its unique positive root, we then have (cf. (4.5))

$$
\left|z_{i}\right| \leqq \alpha,
$$

$$
(i=1,2, \cdots, n)
$$

similarly,

$$
\left|\omega_{i}\right| \leqq \alpha, \quad(i=1,2, \cdots, k-n) .
$$

Therefore, by (5.2), we get

$$
\left|a_{n}\right|=\left|z_{1}\right| \cdot\left|z_{2}\right| \cdots\left|z_{n}\right| \leqq \alpha^{n} .
$$

The right equality sign in (5.4) cannot hold, since $L(\alpha) \geqq 3$. Thus, the left inequality of (2.1) holds.

From (5.1), 


$$
\alpha^{k}-\alpha^{j}=r=\left|z_{1}\right| \cdot\left|z_{2}\right| \cdots\left|z_{n}\right| \cdot\left|\omega_{1}\right| \cdot\left|\omega_{1}\right| \cdots\left|\omega_{k-n}\right|,
$$

and therefore, by (5.3),

$$
\alpha^{k}-\alpha^{j} \leqq\left|a_{n}\right| \alpha^{k-n} \text {. }
$$

The right inequality of (2.1) is simply a rearrangement of (5.5). This completes the proof of Lemma 5.1 .

REMARK 1. We show that the equality sign in (2.1) holds if and only if

$$
M_{\alpha}(x)=x^{k}-x^{j}-\left|a_{k}\right| \text {. }
$$

Sufficiency. Assume that $M_{\alpha}(x)=x^{k}-x^{j}-\left|a_{k}\right|$. Then

$$
\frac{\alpha^{n}-\left|a_{n}\right|}{\alpha^{n}}=\frac{\alpha^{k}-\left|a_{k}\right|}{\alpha^{k}}=\frac{\alpha^{j}}{\alpha^{k}}=\frac{1}{\alpha^{k-j}} .
$$

Necessity. Assume that

$$
\frac{\alpha^{n}-\left|a_{n}\right|}{\alpha^{n}}=\frac{1}{\alpha^{k-j}} .
$$

Since $(j, k)$ is an exponent class, $\alpha^{k}-\alpha^{j}=r$ (a positive integer), where $k \geqq n$. Now,

$$
\frac{\alpha^{n}-\left|a_{n}\right|}{\alpha^{n}}=\frac{\alpha^{3}}{\alpha^{k}}=\frac{\alpha^{k}-r}{\alpha^{k}} .
$$

Equating the first and third expressions in (5.6), we obtain

$$
\left|a_{n}\right| \alpha^{k}=r \alpha^{n} \text {. }
$$

If $k \neq n, \alpha^{k-n}=r /\left|a_{n}\right|$, contradicting the hypothesis that $L(\alpha) \geqq 3$ (cf. §6, Lemma 6.1). Thus $n=k$, and from (5.7), $r=\left|a_{n}\right|$. Therefore $M_{\alpha}(x)=x^{k}-x^{j}$ $-\left|a_{k}\right|$.

Proof of Corollary 5.1. If $g / \alpha$ were not unitary, we would have

$$
0<\alpha^{n}-\left|a_{n}\right|=-a_{1} \alpha^{n-1}-a_{2} \alpha^{n-2}-\cdots-a_{n-1} \alpha<0,
$$

which is impossible.

Proof of Corollary 5.2. We need prove only the left inequality. Thus, if $(j, k)$ is a binary class, with $j<k$,

$$
\alpha^{n-1}(\alpha-1) \leqq \alpha^{n}-\alpha^{n-(k-j)} \leqq\left|a_{n}\right|,
$$

by (2.1).

Proof of Corollary 5.3. The right inequality is the same as (5.2). To prove the left inequality, we have

$$
\begin{aligned}
\alpha^{n-1}(\alpha-1) & \leqq\left|a_{n}\right| \\
& =\left|z_{1}\right| \cdot\left|z_{2}\right| \cdots\left|z_{n}\right| \\
& \leqq \alpha^{n-1}\left|z_{i}\right| .
\end{aligned}
$$


Dividing by $\alpha^{n-1}$, we obtain the desired result.

REMARK 2. The following example shows that the left equality sign in (2.3) may hold for some of the conjugates of certain $\alpha$ :

$$
M_{\alpha}(x)=x^{2}-x-K
$$

$(K>0)$ has $\alpha$ and $1-\alpha(\alpha>1)$ as roots.

6. Proof of Theorem 3. We first prove

LEMмA 6.1. If there exists a positive integer $t$ such that $\alpha^{t}$ is rational, then $L(\alpha)=2$.

Proof. Let $h$ denote the smallest positive integer such that $\alpha^{h}$ is rational; let $\alpha^{h}=v$. Since $\alpha$ is an algebraic integer, $v$ must be integral. We will show that $M_{\alpha}(x)=x^{h}-v$, by proving that the binomial $x^{h}-v$ is irreducible.

If $x^{h}-v$ were reducible, then $v=b^{c}$ (cf. [4]), where $b, c$ are positive integers, $c>1$, and $c$ divides $h$. But, letting $h=r c$,

$$
v=\alpha^{h}=\alpha^{r c}=b^{c} .
$$

This implies $\alpha^{r}=b$, contradicting the definition of $h$.

Lemma 6.2. Let $L(\alpha) \geqq 3$. Suppose that each of the pairs $(j, k),\left(j^{\prime}, k^{\prime}\right)$ forms a binary class of $g / \alpha\left(j<k, j^{\prime}<k^{\prime}\right)$. Then,

$$
k-j \neq k^{\prime}-j^{\prime} \text {. }
$$

Proof. Assume that $k-j=k^{\prime}-j^{\prime}$. Take $k<k^{\prime}$, and let

$$
t=k^{\prime}-k=j^{\prime}-j \text {. }
$$

Then,

$$
\alpha^{t}\left(\alpha^{k}-\alpha^{j}\right)=\alpha^{k^{\prime}}-\alpha^{j^{\prime}}
$$

or,

$$
\alpha^{t}=\frac{\alpha^{k^{\prime}}-\alpha^{j^{\prime}}}{\alpha^{k}-\alpha^{j}}=\frac{u}{v}
$$

where $u, v$ are positive integers. Consequently $L(\alpha)=2$, by Lemma 6.1, contradicting the hypothesis.

Proof of Theorem 3. We may assume that $L(\alpha) \geqq 3$, in view of Theorem 1 .

Let $(j, k)$ be any binary class of $g / \alpha(j<k)$. The right inequality of $(2.1)$ implies that

$$
k-j \leqq n-\log _{\alpha}\left(\alpha^{n}-\left|a_{n}\right|\right) .
$$

Denoting by $N$ the greatest integer less than or equal to the right member of (6.2), we thus have

$$
k-j \leqq N
$$


Thus, by Lemma 6.2 , there are at most $N$ binary classes.

REMARK 1. If $L(\alpha) \geqq 3$, then $g / \alpha^{p}$ is unitary for each positive integer $p$ satisfying:

$$
p>\left[n-\log _{\alpha}\left(\alpha^{n}-\left|a_{n}\right|\right)\right]=N .
$$

Proof. If this statement were false, there would exist a positive integer $p_{0}>N$, and positive integers $r, s(r<s)$ such that

$$
\left(\alpha^{p_{0}}\right)^{s}-\left(\alpha^{p_{0}}\right)^{r}
$$

is integral. Thus the integers $\left(p_{0} r, p_{0} s\right)$ form a binary class for $\mathscr{g} / \alpha$. But,

$$
p_{0} s-p_{0} r=p_{0}(s-r)>N
$$

contradicting (6.3).

REMARK 2 . If $g / \alpha$ is unitary, then $g / \alpha^{p}$ is also unitary for all integers $p \geqq 1$.

7. Proof of Theorem 4. Let $C_{0}$ be any exponent class of $g / \alpha$, and let $m$ denote its smallest element. We show that no integer larger than $m$ can belong to $C_{0}$.

Let the canonical form of $\alpha^{m}$ (i.e. $\alpha^{m}$ expressed in terms of the basis $\left\{1, \alpha, \alpha^{2}, \cdots, \alpha^{n-1}\right\}$ with integral coefficients) be given by

$$
\alpha^{m}=c_{1} \alpha^{n-1}+c_{2} \alpha^{n-2}+\cdots+c_{n-1} \alpha+c_{n},
$$

where each $c_{i} \geqq 0$, since each $b_{i} \geqq 0$. Now, for each positive integer $t$, we shall denote by $S_{t}$ the sum of all the coefficients in the canonical form of $\alpha^{t}$, excluding the "constant" (i.e. $\alpha$-free) term, if any. Since (7.1) yields

$$
\alpha^{m+1}=\left(c_{1} b_{1}+c_{2}\right) \alpha^{n-1}+\left(c_{1} b_{2}+c_{3}\right) \alpha^{n-2}+\cdots+\left(c_{1} b_{n-1}+c_{n}\right) \alpha+c_{1} b_{n},
$$

we obtain

$$
S_{m+1}=S_{m}+c_{n}+c_{1}\left(\left(\sum_{i=1}^{n-1} b_{i}\right)-1\right) .
$$

CASE I. Assume that at least one of the integers $c_{1}, c_{n}$ is positive. Then, from (7.2), $S_{m+1}>S_{m}$. Moreover, since $S_{t}$ is clearly a nondecreasing function of $t$, we conclude that $S_{t}>S_{m}$ for each $t>m$. Thus, if $t>m, \alpha^{t}$ cannot have the same canonical form (excluding the "constant" term) as $\alpha^{m}$. Therefore, it is impossible that $\alpha^{t}=\alpha^{m}+K$, where $K$ is a positive integer.

CASE II. $c_{1}=c_{n}=0$. Let $c_{q}$ be the first nonzero coefficient in (7.1). We then have

$$
\begin{aligned}
\alpha^{m} & =c_{q} \alpha^{n-q}+c_{q+1} \alpha^{n-q-1}+\cdots+c_{n-1} \alpha, \\
\alpha^{m+1} & =c_{q} \alpha^{n-q+1}+c_{q+1} \alpha^{n-q}+\cdots+c_{n-1} \alpha^{2}, \\
& \cdot \cdot \cdot \cdot \cdot \cdot \cdot \cdot \cdot \cdot \cdot \\
\alpha^{m+q-1} & =c_{q} \alpha^{n-1}+c_{q+1} \alpha^{n-2}+\cdots+c_{n-1} \alpha^{q} .
\end{aligned}
$$


Since the above canonical forms are of ascending degrees, it follows that none of the integers

$$
m+1, m+2, \cdots, m+q-1
$$

belongs to $C_{0}$. Now, just as we obtained (7.2), we can write:

$$
S_{m+q}=S_{m+q-1}+c_{q}\left(\left(\sum_{i=1}^{n-1} b_{i}\right)-1\right) .
$$

But, $S_{m+q-1}=S_{m}$, from the first and last equations of (7.3). Thus, $S_{m+q}>S_{m}$, since $c_{q}>0$. Hence, as before, no integer larger than $m$ can belong to $C_{0}$.

8. Proof of Theorem 5. Assume that there exists a binary $\operatorname{class}(j, k)$; that is,

$$
\alpha^{k}-\alpha^{j}=r,
$$

where $r$ is a positive integer. But, since the constant term of $M_{\alpha}(x)$ is -1 , the product of its roots is \pm 1 . Hence, since $\alpha>1$, it follows that $M_{\alpha}(x)$ must have a root $\beta$ such that $|\beta|<1$. We then conclude, as in the proof of Lemma 4.1 , that $r=1$. From (8.1),

$$
x^{k}-x^{j}-1=M_{\alpha}(x) \cdot P(x),
$$

where $P(x)$ is a polynomial with integral coefficients. Letting $x=1$,

$$
P(1)=-\frac{1}{M_{\alpha}(1)}
$$

Therefore, since $P(1)$ must be integral, it follows that $M_{\alpha}(1)= \pm 1$. Now, $M_{\alpha}(1)$ cannot be positive; otherwise $M_{\alpha}(x)$ would have a root between 0 and 1 , whereas the left member of (8.2) has only one positive root, namely $\alpha$.

We thus conclude that

$$
\sum_{i=1}^{n-1} a_{i}=M_{\alpha}(1)=-1,
$$

which contradicts the hypothesis. This completes the proof.

9. Proof of Theorem 6. It is sufficient to show that $R(x)=x^{k}-x^{j}-r$ cannot have two zeros of the same sign for any pair of values of $j, k$ with $0<j<k$. Since $R(x)$ has one variation in sign, $R(x)$ has exactly one positive zero. If $k$ is even, $R(-x)$ has one variation in sign and therefore $R(x)$ has exactly one negative zero. If $k$ is odd, $R(x)<-r<0$ when $x<-1$ and $R(x)<-x^{j}-r<0$ when $-1<x<0$, so that $R(x)$ has no negative zeros in this case.

10. Proof of Theorem 7. If $a<-1$, it follows from Theorem 4 that $g / \alpha$ is unitary. If $a>0$, it follows from Corollary 5.1 that $g / \alpha$ is unitary. This proves part (a).

Proof of Theorem 7(b). In this case, we are concerned with a minimal polynomial of the form 


$$
M_{\alpha}(x)=x^{n}-x^{n-r}-K,
$$

where $K>0$. Thus, the integers $n-r$ and $n$ form a binary class, which we shall refer to as the trivial binary class. When (10.1) is of the special form

$$
M_{\alpha}(x)=x^{3 t}-x^{t}-1,
$$

the existence of the nontrivial binary class $(4 t, 5 t)$ follows from the identity

$$
\left(x^{5 t}-x^{4 t}-1\right)=\left(x^{3 t}-x^{t}-1\right)\left(x^{2 t}-x^{t}+1\right) .
$$

In order to complete the proof of Theorem $7(b)$, we must show that the class $(4 t, 5 t)$, associated with the minimal polynomial $(10.2)$, is the only case of a nontrivial binary class arising from a minimal polynomial of the form (10.1).

We begin by establishing

Lemma 10.1. Suppose that $M_{\alpha}(x)$ is of the form (10.1). Then, the positive integer $p$ will be the smaller element of a nontrivial binary class if and only if the canonical form of $\alpha^{p}$ is of type

$$
\alpha^{p}=c \alpha^{n-q}+c \alpha^{n-2 q}+c \alpha^{n-3 q}+\cdots+c \alpha^{n-m q},
$$

where $c>0, m q=r, m>1$. Moreover, the larger element of this binary class must $b e(p+q)$.

Proof. The sufficiency is immediate, since (10.4) implies

$$
\begin{aligned}
\alpha^{p+q} & =c \alpha^{n-q}+c \alpha^{n-2 q}+\cdots+c \alpha^{n-m q}+c K \\
& =\alpha^{p}+c K .
\end{aligned}
$$

For the necessity, we assume that $p$ is the smaller element of a nontrivial binary class $C_{0}$, and denote the canonical form of $\alpha^{p}$ by

$$
\alpha^{p}=c_{q} \alpha^{n-q}+c_{q+1} \alpha^{n-q-1}+c_{q+2} \alpha^{n-q-2}+\cdots+c_{n-1} \alpha+c_{n},
$$

all $c_{i} \geqq 0, c_{q}>0, q \geqq 1$. We note that $c_{n}=0$; otherwise, as in the proof of Theorem 4 , Case I, we would have: $S_{t}>S_{p}$, for each $t>p$, so that $p$ could not be the smaller element of a binary class.

From (10.5), we see that $\alpha^{p+1}, \alpha^{p+2}, \cdots, \alpha^{p+q-1}$ will each have canonical forms of degree higher than $(n-q)$, so that none of the integers $p+1, p+2$, ..., $p+q-1$ can belong to $C_{0}$. Moreover, since $\alpha^{p+q}$ will contain the "constant" term $c_{q} K$ in its canonical form, no integer larger than $(p+q)$ can belong to $C_{0}$ (cf. Theorem 4, Case I). Thus, the second element of $C_{0}$ must be $(p+q)$.

By the "pure-canonical" form of $\alpha^{w}$ ( $w$ integral), we shall mean the canonical form of $\alpha^{w}$ with all zero terms omitted. We note that the pure-canonical form of $\alpha^{w}(w \geqq 0)$ has all positive coefficients if $M_{\alpha}(x)$ is of the form (10.1).

We next show that each exponent in the pure-canonical form of $\alpha^{p}$ must 
be of form $(n-a q)$, where " $a$ " is a positive integer. Assume that this is not the case, and let $u$ be the largest exponent which is not of this form. Suppose that $u$ falls between $(n-b q)$ and $(n-(b+1) q)$, where $b$ is a positive integer. But then, $\alpha^{p+q}$ would contain the exponent $(u+q)$ in its pure-canonical form, while $\alpha^{p}$ does not, which is impossible.

Moreover, since $\alpha^{p+q}$ contains the exponent $(n-r)$ in its pure-canonical form, so must $\alpha^{p}$; hence, $r=m q, m \geqq 1$. Thus (10.5) becomes

$$
\alpha^{p}=c_{q} \alpha^{n-q}+c_{2 q} \alpha^{n-2 q}+\cdots+c_{m q} \alpha^{n-m q}+\cdots+c_{v q} \alpha^{n-v q},
$$

where $v \geqq m, c_{m q}>0, c_{v q}>0$.

We next see that $v=m$. For, if $v>m, \alpha^{p+q}$ would not contain the exponent $(n-v q)$ in its pure-canonical form. Thus, (10.6) becomes

$$
\alpha^{p}=c_{q} \alpha^{n-q}+c_{2 q} \alpha^{n-2 q}+\cdots+c_{m q} \alpha^{n-m q},
$$

where $m q=r$. We can now see that $m>1$; otherwise, we would have

$$
\alpha^{p}=c_{q} \alpha^{n-r}, \text { or } \alpha^{p-n+r}=c_{q},
$$

contradicting Lemma 6.1.

Finally, from (10.7) we obtain

$$
\alpha^{p+q}=c_{2 q} \alpha^{n-q}+C_{3 q} \alpha^{n-2 q}+\cdots+c_{m q} \alpha^{n-(m-1) q}+c_{q} \alpha^{n-m q}+c_{q} K .
$$

Comparing (10.8) and (10.7), we conclude that

$$
c_{q}=c_{2 q}=\cdots=c_{m q},
$$

completing the proof of Lemma 10.1 .

It will now be shown that if there exists an integer $p$ such that $\alpha^{p}$ is of form (10.4), then the minimal polynomial (10.1) must be of form (10.2), where $p=4 t, q=t$. Toward this end, we first establish:

LEMMA 10.2. If there exists an integer $p$ such that $\alpha^{p}$ is of form (10.4), then $K=1$ in (10.1), and $c=1$ in (10.4).

Proof. Dividing (10.4) by $\alpha^{n-m q}$, we have

$$
\alpha^{p-n+m q}=c\left[\alpha^{(m-1) q}+\alpha^{(m-2) q}+\cdots+\alpha^{q}+1\right] .
$$

Therefore,

$$
\begin{aligned}
x^{p-n+m q}-c\left[x^{(m-1) q}+x^{(m-2) q}+\cdots+x^{q}\right. & +1] \\
& =\left(x^{n}-x^{n-r}-K\right) \cdot P(x),
\end{aligned}
$$

where $P(x)$ is a polynomial with integral coefficients. Letting $x=0$ in (10.10), we get: $-c=-K \cdot P(0)$, so that $K$ divides $c$. Then letting $x=1$ in (10.10), we get: $1-m c=-K \cdot P(1)$, so that $K$ also divides $m c-1$. Since $m>1, m c$ $-1 \neq 0$; it thus follows that $K=1$.

Since $K=1, \alpha$ is a unit in the ring $H$ of algebraic integers. Therefore, 
$\alpha^{p-n+m q}$ is also a unit. But, according to (10.9), $c$ must divide $\alpha^{p-n+m q}$ (in $H$ ), since the quantity in the bracket is an algebraic integer. Hence, $c$ is a unit, and must be 1 .

This completes the proof of Lemma 10.2.

Lemma 10.3. If $\alpha^{p}$ has a canonical form of type (10.4), then $n<p<n+r$.

Proof. It is clear that $n<p$; otherwise, $\alpha$ would satisfy an equation of degree less than $n$.

Now, since $K=1,(10.1)$ becomes

$$
\alpha^{n}=\alpha^{n-r}+1 .
$$

Therefore,

$$
\alpha^{n+r}=\alpha^{n-r}+\alpha^{r}+1 .
$$

Suppose first that $n+r \leqq p<2 n$. That is, $p=n+r+s$, where $0 \leqq s<n-r$. The canonical form of $\alpha^{p}$ can then be obtained by multiplying (10.12) by $\alpha^{s}$. Therefore, the terminating exponent in the pure-canonical form of $\alpha^{p}$ will be less than $n-r$, contrary to (10.4).

Next, suppose $p \geqq 2 n$. Squaring (10.11),

$$
\alpha^{2 n}=\alpha^{2(n-r)}+2 \alpha^{n-r}+1 .
$$

Thus, the canonical form of $\alpha^{p}$ will have at least one coefficient $\geqq 2$, since $\alpha^{p}=\alpha^{p-2 n} \cdot \alpha^{2 n}$ and all coefficients in the canonical form of $\alpha^{p-2 n}$ are nonnegative integers. But this contradicts (10.4), since $c=1$.

This concludes the proof of Lemma 10.3.

Lemma 10.4. If there exists an integer $p$ such that $\alpha^{p}$ is of form (10.4), then $M_{\alpha}(x)$ must be of form (10.2), where $p=4 t$ and $q=t$.

Proof. By Lemma 10.3, we have: $p=n+t$, where $0<t<r$. From (10.11), we obtain

$$
\alpha^{p}=\alpha^{n-(r-t)}+\alpha^{t} .
$$

Comparing (10.14) with (10.4), we conclude that $r-t=q$, and $t=n-r$ $=n-2 q$. These relations imply that $q=t, n=3 t, r=2 t$, so that (10.1) becomes (10.2). Moreover, $p=n+t=4 t$.

The proof of Theorem 7 is now complete.

11. A class of $M_{\alpha}(x)$ with nonunitary $\mathscr{I} / \alpha$ and $L(\alpha)>3$. Consider the class of polynomials of form

$$
P(x)=x^{(2 r-1) q}-2 x^{(2 r-2) q}+2 x^{(2 r-3) q}-\cdots+2 x^{q}-2,
$$

where $r$ and $q$ are positive integers, $r \geqq 2$. We first note that $P(x)$ is irreducible by Eisenstein's Criterion (cf. [5]). Moreover, from the identity

$$
\left(x^{q}+1\right) \cdot P(x)=x^{2 r q}-x^{(2 r-1) q}-2,
$$


we see that $P(x)$ has exactly one positive root $\alpha(1<\alpha<2)$. Thus $P(x)$ is the minimal polynomial of $\alpha$. Finally, from (11.1), we see that $(2 r-1) q$ and $2 r q$ form a binary class of $g / \alpha$.

12. Some unanswered questions. The decomposition $g / \alpha$ has at most a finite number of binary classes, by Theorem 3. However, the authors have no example of an $\alpha$ for which $g / \alpha$ has more than two binary classes; nor do they have an example for which $g / \alpha$ has exactly two binary classes, aside from the case where $M_{\alpha}(x)$ is of the form $x^{3 t}-x^{t}-1$ (cf. Theorem 7).

We therefore pose the following questions:

(1) Does there exist an $\alpha$ for which $g / \alpha$ has more than two binary classes?

(2) Does there exist an $\alpha$, other than the case where $M_{\alpha}(x)$ is of the form $x^{3 t}-x^{t}-1$, for which $g / \alpha$ has exactly two binary classes?

We note that if there exists a $t_{0}$ such that $Q(x)=x^{3 t_{0}}-x^{t_{0}}-1$ is reducible, then the positive root $\alpha$ of $Q(x)$ will induce a decomposition $g / \alpha$ having at least two binary classes: $\left(t_{0}, 3 t_{0}\right),\left(4 t_{0}, 5 t_{0}\right)$ (cf. (10.3)). Furthermore, for this case, $M_{\alpha}(x)$ cannot be of the form $x^{3 s}-x^{s}-1\left(s<t_{0}\right)$; otherwise, $g / \alpha$ would have at least four binary classes: $(s, 3 s),(4 s, 5 s),\left(t_{0}, 3 t_{0}\right),\left(4 t_{0}, 5 t_{0}\right)$, contradicting Theorem 7.

\section{REFERENCES}

1. J. F. Koksma, Ein mengentheoretischer Satz über die Gleichverteilung modulo Eins, Compositio Math. vol. 2 (1935) pp. 250-258.

2. E. Hecke, Vorlesungen über die Theorie der algebraichen Zahlen, New York, Chelsea, 1948. cf. Satz 60 p. 78; or H. Pollard, The theory of algebraic numbers, New York, Wiley, 1950. Cf. Lemma 6.1, p. 58.

3. A. Gelfond, Sur la divisibilité de la différence des puissances de deux nombres entiers par une puissance d'un idéal premier, Mat. Sb. N.S. vol. 7 (1940) pp. 7-25. Cf. Theorem IV, p. 21.

4. K. Th. Vahlen, Über reductible Binome, Acta Math. vol. 19 (1895) pp. 195-198.

5. B. L. van der Waerden, Modern algebra, vol. 1, New York, Ungar, 1949, p. 74.

City College,

NEw YORK, NEw YORK 